\title{
Burnout, Depression, Anxiety, and Stress Among Family Physicians in Kansas Responding to the COVID-19 Pandemic
}

\author{
Samuel Ofei-Dodoo, PhD, MPA, MA, Colleen Loo-Gross, MD, MPH, and \\ Rick Kellerman, $M D$
}

Introduction: COVID-19 has spread rapidly, with vast global implications. This study assessed how family physicians in Kansas were responding to COVID-19 and the effects of the pandemic on their well-being.

Metbods: The authors conducted a cross-sectional survey of 113 family physicians in Kansas between May 22, 2020 and June 25, 2020. The study participants completed an anonymous, 36-item survey assessing their concerns about being exposed to COVID-19 and levels of personal depression, anxiety, stress, and burnout in addition to demographic information.

Results: There was a $45.6 \%$ response rate, with $50.4 \%(n=57)$ of the respondents reporting manifestations of burnout. The physicians who personally treated any presumptive or confirmed CoviD-19 patient, compared with those who did not, were more likely to report at least 1 manifestation of burnout, experience emotional exhaustion, and feel a higher level of personal stress.

Conclusion: Our findings demonstrate that the COVID-19 pandemic may be taking an emotional toll on family physicians in Kansas. This study provides a baseline from which to continue further monitoring of outcomes. Data can help drive initiatives at local, state, and national levels to help diminish the negative impact of the COVID-19 pandemic on physicians. (J Am Board Fam Med 2021;34:522-530.)

Keywords: CoVID-19, Cross-Sectional Studies, Family Medicine, Family Physicians, Kansas, Mental Health, Occupational Burnout, Pandemics, Surveys and Questionnaires

\section{Introduction}

Since its introduction into the human population in late 2019, the novel coronavirus infectious disease (COVID-19) has spread rapidly, with vast global implications. The outbreak was designated a pandemic by the World Health Organization (WHO) on March 11, 2020. ${ }^{1}$ By November 24, 2020, over 58 million total confirmed cases and 1,388,528 total deaths had been reported globally, with the United States alone accounting for 12,175,921 confirmed

This article was externally peer reviewed.

Submitted 6 October 2020; revised 28 November 2020; accepted 30 November 2020.

From the Department of Family and Community Medicine, University of Kansas School of Medicine-Wichita (SO-D, CL-G, RK).

Funding: None.

Conflict of interest: None.

Corresponding author: Samuel Ofei-Dodoo, PhD, MPA, MA, Department of Family and Community Medicine, University of Kansas School of Medicine-Wichita, 1010 N. Kansas, Wichita, KS 67214 (E-mail: sofeidodoo@kumc. edu). cases and 255,958 deaths. ${ }^{2,3}$ The number of cases began to rise sharply in Midwestern states during November, affecting more small towns and rural areas, with daily case rates surpassing 4600 per 100,000 population in the state of Kansas, compared with just over 2000 per 100,000 at the beginning of October $2020 .^{3}$ As the world continues to struggle with the widespread infection, it is important to consider the short-term and long-term effects on the health care system and workforce.

Before the COVID-19 pandemic, the risk of burnout and stress-related problems such as depression and anxiety in physicians had been identified as an important area of public health concern. As compared with the general population, physicians are at higher risk of experiencing symptoms of burnout as well as dissatisfaction with work-life balance. ${ }^{4}$ Among different specialties, physicians practicing family medicine tend to have higher rates of burnout symptoms, along with those in emergency medicine and internal medicine. ${ }^{4}$ In addition, 
burnout among family physicians has been shown to have notable variation by state, indicating the need to further assess trends in specific regions. ${ }^{5}$ In Kansas, previous literature has identified the significant presence of burnout in the rural practice setting, which is notable given the geographic nature of the state. ${ }^{6}$ Nearly one half of Kansas physicians experience symptoms of burnout, such as emotional exhaustion, cynicism, and reduced professional efficiency, and are more likely to report thoughts of suicidal ideation, screen positive for depression, and report high degrees of fatigue..$^{7,8}$

The implications of burnout in clinicians are well documented. Physicians with burnout are more likely to experience motor vehicle accidents, mood disorders, and substance and alcohol abuse. Furthermore, studies have shown that burnout is associated with an increased risk of medical errors and malpractice, with decreases in quality of care, productivity, and patient satisfaction. ${ }^{10,11}$ Health care professionals experiencing burnout and other forms of emotional distress have a stronger intention of leaving the medical profession via early retirement and/or career change. ${ }^{12,13}$ Physician wellness has become an increasingly vital topic given both the personal and professional implications.

Health care professionals, including those in primary care specialties such as family medicine, are among those working the front lines in response to the COVID-19 pandemic. Previous literature has shown the association of infectious disease outbreaks with adverse effects on the mental health of health care workers. ${ }^{14-16}$ Initial studies have shown that this concern has emerged with the COVID-19 outbreak, and additional research is warranted given the unprecedented, enduring nature of this pandemic. ${ }^{17-19}$ Primary care physicians are at risk of encountering additional stressors given the significant changes to clinical practice as a result of COVID-19, such as the financial impact associated with decreases in routine medical visits and shifts to telemedicine. Given that the global community continues to be affected by the worsening spread of COVID-19, it is prudent to assess the effects on physician well-being and to ensure appropriate programs are in place to provide emotional, mental health, and social support for health care workers. The purpose of this study was to assess the effects of the COVID-19 pandemic on burnout and other types of emotional distress among family physicians in Kansas, many of whom are members of the University of Kansas School of
Medicine Department of Family and Community Medicine's practice-based research network. These family physicians work collaboratively through research and quality improvement activities to improve the health of Kansans.

\section{Methods \\ Study Design}

The study was a cross-sectional survey of family physicians in Kansas. The authors surveyed community-based family physicians as well as faculty physicians in Kansas. The questionnaire focused on how family physicians in Kansas were responding to the COVID-19 pandemic at the time of the study from May 22 to June 25, 2020. The University of Kansas School of Medicine-Wichita (KUSM-W) Institutional Review Board granted exemption for the study.

\section{Study Instrument}

We used a 36-item questionnaire (see the Appendix) to assess family physicians' concerns about being exposed to COVID-19 and levels of burnout and personal depression, anxiety, and stress. The questionnaire included items on demographic information (age, gender, years in clinical practice, and the Kansas county where the physicians primarily practiced), whether the physicians had treated presumptive or confirmed COVID-19 patients, the number of presumptive or confirmed COVID-19 patients treated, place (office, home visits, emergency department, hospital, long-term care facility, telehealth) the COVID19 patients were treated, and patient volumes before and during the early phase of the pandemic.

\section{Burnout}

Manifestations of burnout among the respondents were assessed using 2 single-item measures of emotional exhaustion and depersonalization adapted from the full Maslach Burnout Inventory (MBI-22), which have been previously validated. ${ }^{20}$ The emotional exhaustion item ("I feel burnout from my work") and depersonalization item ("I've become more callous toward people since I became a physician") have been shown to be useful screening questions for burnout. ${ }^{21-23}$ These 2 items have shown the highest factor loading ${ }^{20,24,25}$ and strongest correlation ${ }^{21,26}$ with their respective emotional exhaustion and depersonalization domains in the MBI$22 .^{21}$ The 2 single items have been used in previous 
studies to measure emotional exhaustion, depersonalization, and manifestations of burnout among physicians. ${ }^{4,26-29}$ The physicians recorded the degree to which each item applied to themselves on a 7 -point Likert scale $(0=$ never, $6=$ every day). The scores of each item were classified into low, moderate, and high burnout categories using established cutoffs. 4,20,27,29 Higher scores are indicative of greater exhaustion and depersonalization and higher levels of burnout. Consistent with convention, ${ }^{4,27,29}$ we considered physicians who scored high (score of greater than 3) on exhaustion and/or depersonalization domains as having at least 1 manifestation of professional burnout.

\section{Depression, Anxiety, and Stress}

We used the Depression Anxiety Stress Scales-21 (DASS-21) to measure the respondents' emotional state. DASS-21 is a validated research tool that has been used widely to assess quality of life and consists of 21 questions in 3 scales designed to measure negative emotional states of depression, anxiety, and stress. ${ }^{30,31}$ These scales have been found to have high internal consistency and can be used in a variety of settings to measure an individual's current emotional state and changes over time. ${ }^{32}$ Participants recorded how much a statement applied to them over the past week on a 4-point Likert scale $(0=$ never, $3=$ almost always $)$. Scores for the 7 questions specific to each of the 3 scales were summed with a possible score ranging from 0 to 21. Higher scores indicate greater levels of the corresponding emotional state.

We also assessed the physicians' self-reported stress surrounding COVID-19 using the following 4 questions: "What is your level of concern about personally being exposed to COVID-19 because of your work?"; "What is your level of concern about exposing family members to COVID-19 because of your work?"; "What was your level of stress before the COVID-19 pandemic?"; and "What is your level of personal stress as a result of the COVID-19 pandemic?"

\section{Data Collection Process}

The questionnaire was hosted in SurveyMonkey, a secure web-based survey system. A generated link to the 36-item questionnaire was sent via e-mail to potential participants. The Department of Family and Community Medicine (DFCM) of the KUSMW uses an e-mail system called FM-RADIO (Family Medicine Research and Data, Information and
Outcomes Practice-Based Research Network) as a survey collection tool. The FM-RADIO is an electronic practice-based research network composed of actively practicing family physicians throughout the state of Kansas who are KUSM-W family medicine residency program graduates, family physician nonKUSM-W graduates, faculty physicians, and resident physicians. The link to the survey was sent only to the 248 actively practicing community physicians and KUSM-W family medicine faculty physicians who were on the FM-RADIO list. Participation was voluntary, and responses were anonymous. We later sent 2 reminders to those who had not completed the survey. Data collection occurred between May 22 and June 25, 2020. No compensation was provided to participants.

\section{Statistical Analyses}

Standard descriptive statistics were used to create a demographic profile and describe the levels of personal depression, anxiety, stress, and burnout among the physicians. Mann-Whitney $U$ test/independent samples $t$-test (for continuous variables) and likelihood ratio chi square (for categorical variables) were used to evaluate associations between variables. Generalized linear mixed models were used to calculate associations between the responses to the question "Have you cared for presumptive or confirmed COVID-19 patients in your practice?" modeled as a binary outcome (yes/no) against a single fixed effect for independent variables (DASS21, burnout, emotional exhaustion, depersonalization, age, gender, and years in clinical practice).

Adjusted odds ratios were estimated by modeling all significant independent variables against the responses to the question "Have you cared for presumptive or confirmed COVID-19 patients in your practice?" controlling for physicians' age, gender, and years in clinical practice. A sample size of 100 was calculated as necessary for adequate power $(>0.85)$ to detect significant relationships among the variables with 1 degree of freedom, $P<.05$, and 0.5 effect size. ${ }^{33,34}$ All analyses were 2 -sided with $\alpha$ of 0.05 . The IBM SPSS (Statistical Package for the Social Sciences), version 26 was used for these analyses.

\section{Results}

\section{Respondent Characteristics}

The response rate was $45.6 \%$ (113/248). Table 1 represents the demographic information of the 
Table 1. Respondents' Characteristics $(n=113)$

\begin{tabular}{lc}
\hline Characteristics & All Respondents \\
\hline Gender, no. (\%) & \\
Male & $53(46.9)$ \\
Female & $43(38.1)$ \\
Prefer to not answer & $1(0.9)$ \\
Missing* & $16(14.2)$ \\
Age & $(\mathrm{n}=95)$ \\
Mean (SD), y & $48.4(11.9)$ \\
Median & 49 \\
Minimum & 28 \\
Maximum & 70 \\
Year in clinical practice & $(\mathrm{n}=88)$ \\
Mean (SD), y & $16.7(11.8)$ \\
Median & 17.5 \\
Minimum & $<1$ \\
Maximum & 44 \\
Kansas county where the physicians primarily & \\
$\quad$ practiced, no. (\%) & \\
Butler & \\
Saline & $5(4.4)$ \\
Sedgwick & $6(5.3)$ \\
All other counties & $39(34.5)$ \\
Missing* & $45(39.8)$ \\
Cared for preventive or confirmed & $18(15.9)$ \\
COVID-19 patients, no. (\%) & \\
Yes & \\
No & \\
\hline & \\
\hline &
\end{tabular}

*The number of participants who completed the survey but did not provide an answer to this specific question.

$\mathrm{SD}$, standard deviation.

respondents. The average age of respondents was $48.4(\mathrm{SD}=11.9)$; $46.9 \%$ were male; $34.5 \%$ indicated practicing primarily in Sedgwick County; all others practiced in 30 counties. Most of the respondents $(80.5 \%)$ reported that they had treated a presumptive or confirmed COVID-19 patient (Table 1). There was a $6.8 \%$ margin of error at a $95 \%$ confidence level between the study sample and the population of all practicing family physicians in Kansas, demonstrating that our sample generally represented the population of the practicing family physicians in Kansas. ${ }^{35}$ As Table 2 shows, there was a wide range (74) of the number of COVID-19 patients who had been treated by family physicians at the time of the study, with an average number of 5.7 $(\mathrm{SD}=10.9)$. About a third of the COVID-19 patients required hospitalization.

\section{Concerns about Patient Volume}

Most of the respondents $(66 \%, \mathrm{n}=74)$ reported seeing fewer patients as the outbreak progressed, $31 \%(\mathrm{n}=35)$ reported seeing about the same number of patients, and only $3 \%(n=3)$ reported seeing more patients. Most of the respondents $(83 \%, n=$ 93) were concerned that some of their patients may be forgoing routine or acute medical care because the patients were afraid of being exposed to COVID-19 in a physician office or other clinical settings.

\section{Concern about Exposure to COVID-19}

On a scale of 0 to 10 , where 10 represents very concerned, the median level of concern about personally being exposed to COVID-19 as a result of their work was 5 (mean $=5.0, \mathrm{SD}=3.0$ ), while the median level of concern about exposing family members to COVID-19 as a result of their work was 6 (mean $=6.4, \mathrm{SD}=2.8$ ).

\section{Burnout, Depression, Anxiety, and Stress}

In aggregate, $50.4 \%(\mathrm{n}=57)$ of all respondents met the criteria for manifestations of burnout. Manifestations of burnout were significantly higher in respondents who personally treated any presumptive or confirmed COVID-19 patients, compared with those who did not personally treat any

Table 2. Number and Places Where COVID-19 Patients Were Treated $(\mathbf{n}=91) *$

\begin{tabular}{lc}
\hline Measure & Participants \\
\hline COVID-19 patients treated & \\
Mean (SD) & $5.7(10.9)$ \\
Median & 2 \\
Minimum & 1 \\
Maximum & 75 \\
Places where COVID-19 patients treated, no. (\%) & \\
In office & $24(26.4)$ \\
At the hospital & $35(38.5)$ \\
Via telehealth & $13(14.3)$ \\
Emergency room & $1(1.1)$ \\
Long-term care facility & $1(1.1)$ \\
Other & $2(2.2)$ \\
Missing &
\end{tabular}

*Table included only physicians who treated COVID-19 patients.

${ }^{+}$The number of participants who completed the survey but did not provide an answer to this specific question.

$\mathrm{SD}$, standard deviation. 
Table 3. Odds Ratios for Independent Variables and Whether Physician Cared for Presumptive or Confirmed COVID-19 ( $\mathrm{n}=113)$

\begin{tabular}{|c|c|c|}
\hline Variables & Odds Ratio $(95 \% \mathrm{CI})$ & $P$ Value \\
\hline \multicolumn{3}{|l|}{ Gender $(\mathrm{n}=95)$} \\
\hline Male vs female & $1.58(0.58$ to 4.32$)$ & $>0.05$ \\
\hline Age (for each additional year older) $(\mathrm{n}=95)$ & $1.01(0.92$ to 1.06$)$ & $>0.05$ \\
\hline Years in clinical practice (for each additional year in practice) $(\mathrm{n}=88)$ & $1.02(0.97$ to 1.06$)$ & $>0.05$ \\
\hline \multicolumn{3}{|l|}{ DASS-21 } \\
\hline Depression $(\mathrm{n}=97)$ & $1.12(0.94$ to 1.34$)$ & $>0.05$ \\
\hline Anxiety $(n=98)$ & $1.23(0.83$ to 1.83$)$ & $>0.05$ \\
\hline Stress $(n=99)$ & $1.13(1.01$ to 1.27$)$ & 0.032 \\
\hline \multicolumn{3}{|l|}{ Manifestations of burnout $(\mathrm{n}=101)$} \\
\hline Burned out versus not burned out & $3.96(1.38$ to 11.36$)$ & 0.011 \\
\hline \multicolumn{3}{|l|}{ Emotional exhaustion $(\mathrm{n}=101)$} \\
\hline High score & $3.21(1.01$ to 10.10$)$ & 0.042 \\
\hline Moderate score & $0.98(0.27$ to 3.60$)$ & $>0.05$ \\
\hline Low score & Reference & - \\
\hline \multicolumn{3}{|l|}{ Depersonalization $(\mathrm{n}=101)$} \\
\hline High score & $2.74(0.82$ to 9.30$)$ & $>0.05$ \\
\hline Moderate score & $2.84(0.57$ to 14.08$)$ & $>0.05$ \\
\hline Low score & Reference & - \\
\hline
\end{tabular}

DASS-21, Depression Anxiety Stress Scales-21.

CI, confidence interval.

presumptive or confirmed COVID-19 patients $\left(63 \%\right.$ vs $30 \% ; \chi^{2}[1, \mathrm{n}=101]=7.1 ; P=.008 ; \mathrm{Phi}=$ $-0.265)$. As Table 3 shows, findings of the mixed model analyses indicated that there was a significant positive association between treating any presumptive or confirmed COVID-19 patients and manifestations of burnout (odds ratio $[\mathrm{OR}]=3.96 ; 95 \% \mathrm{CI}$, $1.38-11.36 ; P=.011)$. In addition, treating presumptive or confirmed COVID-19 patients was associated with high level of emotional exhaustion $(\mathrm{OR}=3.21 ; 95 \% \mathrm{CI}, 1.01-10.10 ; P<.05)$. These associations were not significant after controlling for the respondents' age, gender, and years in clinical practice.

On a scale of 0 to 10 , where 10 represents extreme stress, the respondents reported a median personal stress level of 3 (mean $=3.3, \mathrm{SD}=1.9)$ before the COVID-19 pandemic, compared with a median personal stress level of $6 \quad($ mean $=5$, $\mathrm{SD}=2.3$ ) during the pandemic. The respondents who personally treated any presumptive or confirmed COVID-19 patients reported a median personal stress level of $6 \quad($ mean $=5.3, \mathrm{SD}=2.3)$, compared with a median stress level of 3.5 (mean $=4.1, \mathrm{SD}=2.0$ ) for those who did not.

Stress, as measured by DASS-21, was significantly associated with treating presumptive or confirmed COVID-19 patients $(\mathrm{OR}=1.13 ; 95 \%$ CI, 1.01-1.27; $P=.032$ ). This association remained significant, even after adjusting for respondents' age, gender, and years in clinical practice (adjusted $\mathrm{OR}=1.16 ; 95 \% \mathrm{CI}, 1.01-1.33 ; P=.042$ ).

\section{Discussion}

To our knowledge, this is the first published study of its kind specifically assessing the effect of the COVID-19 pandemic on the emotional health of family physicians in Kansas. The findings of the study showed that half of the family physicians reported at least 1 manifestation of burnout. This finding correlates with previous, pre-COVID-19 research findings where $45.8 \%$ to $49.5 \%$ of practicing physicians in Kansas reported at least 1 symptom of burnout. ${ }^{6-8}$ Our findings show that $80.5 \%$ of this sample of family physicians had treated a presumptive or confirmed COVID-19 patient since the first confirmed case was reported in the United States on January 20, 2020, confirming that family physicians are working on the front lines of the COVID-19 outbreak. ${ }^{36}$ The physicians who personally treated any presumptive or confirmed COVID-19 patients, compared with those who did not, were 4 times more likely to report at least 1 
manifestation of burnout and 3 times more likely to experience emotional exhaustion. The association between the higher level of stress and having personally treated any presumptive or confirmed COVID-19 patient remained significant, even when we adjusted for the physicians' age, gender, and years in clinical practice.

The authors compared self-reported stress levels before and during the pandemic. Responses indicated that personal levels of stress were higher after the onset of the COVID-19 pandemic, though a degree of physician stress was present at baseline. Respondents were more concerned about potential exposure of a family member to the virus than they were concerned about their own personal exposure. Interestingly, the level of concern about family exposure was higher in those who had not treated presumptive or confirmed COVID-19 patients. This difference may be attributed to a sense of anxiety in the setting of an unfamiliar disease and less experience with treatment protocols for these individuals.

The results of this study are consistent with previous studies, which have shown increased stress or other psychological symptoms in those caring directly for presumptive and confirmed COVID-19 patients. ${ }^{17,37,38}$ An earlier study of health care workers in China showed an increase in psychological symptoms among those working on the front lines of the COVID-19 outbreak (eg, depression, anxiety, insomnia, and psychological distress). ${ }^{17} \mathrm{~A}$ previous study of emergency medicine physicians in the United States similarly showed both increased stress levels at home and work as well as increased emotional exhaustion and burnout as a result of the COVID-19 pandemic. $^{37}$

Findings from a nationwide survey have demonstrated higher levels of depression, anxiety, and tiredness, as well as self-perceived COVID-19 related stress, among health care workers compared with the general population. ${ }^{38}$ A study by the Larry A. Green Center and the Primary Care Collaborative found that $63 \%$ of primary care physicians reported feelings of stress, and $80 \%$ reported at least 1 manifestation of professional burnout since the onset of the COVID-19 coronavirus pandemic. ${ }^{39,40}$ Given the increased levels of burnout and other forms of emotional distress among family physicians during the pandemic, there should be improved recognition and development of resources to address the problem. Policy makers and health care organizations can help by recognizing the deleterious effects of emotional distress on physician well-being and ensure appropriate programs are in place to provide emotional, mental health, and social support for health care workers, especially those on the forefront of the pandemic. ${ }^{41}$

\section{Study Limitations}

Our study has limitations. First, although similar to other studies of family physicians practicing in Kansas, ${ }^{42-44}$ the $45.6 \%$ overall response rate limits the generalizability of the findings to active family physicians in Kansas. The cross-sectional nature and inability to establish a direct causal relationship between COVID-19 and respondent distress reduce the generalizability of the results. The instrument relied on self-reported data collected in the form of an online survey and may be influenced by recall and selection biases. The study population was limited to a specific geographic area, though for the purposes of this study, family physicians in Kansas were the target population. Given that organizational support and structure might have an impact on physicians' feelings of stress or anxiety, lack of assessment of the participants' practice setting limits the study findings. Finally, the timing of the study provides data only from the period of data collection, whereas long-term outcomes will likely be affected as the pandemic continues.

Future studies are needed to understand the enduring and long-term effects of the COVID-19 pandemic on physicians as well as other frontline health care workers. A previous study showed an additional increase in stress, anxiety, and depression symptoms among those who had cared for patients with severe acute respiratory syndrome (SARS) 1 year after the peak of the outbreak. ${ }^{14}$ It is reasonable to assume that the extended duration of the current pandemic will continue to be associated with physician burnout, stress, and other psychological symptoms. In addition, future studies that are qualitative in nature would provide a deeper and more nuanced understanding of how COVID-19 impacts primary care clinicians.

\section{Conclusions}

This study provides a baseline from which to continue monitoring of emotional distress, to determine wellness interventions, and to guide development of appropriate physician support systems. These data can help drive wellness and 
mental health support initiatives at local, state, and national levels to help reduce the negative impact of the COVID-19 pandemic on not only physicians but the health care system and workforce overall. It is prudent to address these issues both for the wellbeing of individuals as well as the potential effects on patient care and quality outcomes. This may have an even greater impact in areas of physician shortages, such as the rural communities across Kansas and similar regions globally.

To see this article online, please go to: http://jabfm.org/content/ 34/3/522.full.

\section{References}

1. World Health Organization (WHO). Rolling updates on coronavirus disease (COVID-19). https:// www.who.int/emergencies/diseases/novel-coronavirus2019/events-as-they-happen. Accessed August 28, 2020.

2. World Health Organization (WHO). WHO coronavirus disease (COVID-19) dashboard. https:// covid19.who.int/. Accessed November 24, 2020.

3. Centers for Disease Control and Prevention (CDC). Cases of coronavirus (COVID-19) in the U.S. https://www.cdc.gov/coronavirus/2019-ncov/ cases-updates/cases-in-us.html. Accessed November 24, 2020.

4. Shanafelt TD, Boone S, Tan L, et al. Burnout and satisfaction with work-life balance among US physicians relative to the general US population. Arch Intern Med 2012;172:1377-85.

5. Hansen A, Peterson LE, Fang B, Phillips RL, Jr. Burnout in young family physicians: variation across states. J Am Board Fam Med 2018 Jan-Feb;31:7-8.

6. Marquez-Cunningham D, Lenherr E, Flynn E, Warner J, Zackula R, Cathcart-Rake W. Physician burnout in a rural Kansas community. Kansas Journal of Medicine 2019;12:109-16.

7. Ofei-Dodoo S, Kellerman R, Gilchrist K, Casey EM. Burnout and quality of life among active member physicians of the Medical Society of Sedgwick County. Kansas Journal of Medicine 2019;12:33-9.

8. Ofei-Dodoo S, Ebberwein C, Kellerman R. Assessing loneliness and other types of emotional distress among practicing physicians. Kansas Journal of Medicine 2020;13:1-5.

9. West CP, Dyrbye LN, Shanafelt TD. Physician burnout: contributors, consequences and solutions. J Intern Med 2018;283:516-29.

10. Patel RS, Bachu R, Adikey A, Malik M, Shah M. Factors related to physician burnout and its consequences: a review. Behav Sci (Basel) 2018;8:98.

11. Shirom A, Nirel N, Vinokur AD. Overload, autonomy, and burnout as predictors of physicians' quality of care. J Occup Health Psychol 2006;11: 328-42.

12. Dewa CS, Jacobs P, Thanh NX, Loong D. An estimate of the cost of burnout on early retirement and reduction in clinical hours of practicing physicians in Canada. BMC Health Serv Res 2014;14:254.

13. Soler JK, Yaman H, Esteva M, European General Practice Research Network Burnout Study Group, et al. Burnout in European family doctors: the EGPRN study. Fam Pract 2008;25:245-65.

14. Maunder R, Hunter J, Vincent L, et al. The immediate psychological and occupational impact of the 2003 SARS outbreak in a teaching hospital. CMAJ 2003;168:1245-51.

15. Shah K, Kamrai D, Mekala H, Mann B, Desai K, Patel RS. Focus on mental health during the coronavirus (COVID-19) pandemic: applying learnings from the past outbreaks. Cureus 2020;12:e7405.

16. McAlonan GM, Lee AM, Cheung V, et al. Immediate and sustained psychological impact of an emerging infectious disease outbreak on health care workers. Can J Psychiatry 2007;52:241-7.

17. Lai J, Ma S, Wang Y, et al. Factors associated with mental health outcomes among health care workers exposed to coronavirus disease 2019. JAMA Netw Open 2020;3:e203976.

18. Shanafelt T, Ripp J, Trockel M. Understanding and addressing sources of anxiety among health care professionals during the COVID-19 pandemic. JAMA 2020;323:2133-4.

19. Spoorthy MS, Pratapa SK, Mahant S. Mental health problems faced by healthcare workers due to the COVID-19 pandemic-a review. Asian J Psychiatr 2020;51:102119.

20. Maslach C, Jackson SE, Leiter MP. Maslach Burnout Inventory manual. 3rd ed. Palo Alto, CA: Consulting Psychologists Press; 1996.

21. West CP, Dyrbye LN, Sloan JA, Shanafelt TD. Single item measures of emotional exhaustion and depersonalization are useful for assessing burnout in medical professionals. J Gen Intern Med 2009; 24:1318-21.

22. Rafferty JP, Lemkau JP, Purdy RR, Rudisill JR. Validity of the Maslach Burnout Inventory for family practice physicians. J Clin Psychol 1986;42:488-92.

23. Ofei-Dodoo S, Moser SE, Kellerman R, Wipperman J, Paolo A. Burnout and other types of emotional distress among medical students. Med. Sci Educ 2019;29:1061-9.

24. Kanste O, Miettunen J, Kyngäs H. Factor structure of the Maslach Burnout Inventory among Finnish nursing staff. Nurs Health Sci 2006;8:201-7.

25. Vanheule S, Rosseel Y, Vlerick P. The factorial validity and measurement invariance of the Maslach Burnout Inventory for human services. Stress Health 2007;23:87-91. 
26. West CP, Dyrbye LN, Satele DV, Sloan JA, Shanafelt TD. Concurrent validity of single-item measures of emotional exhaustion and depersonalization in burnout assessment. J Gen Intern Med 2012;27:1445-52.

27. Dyrbye LN, West CP, Satele D, et al. Burnout among U.S. medical students, residents, and early career physicians relative to the general U.S. population. Acad Med 2014;89:443-51.

28. Dyrbye LN, Thomas MR, Massie FS, et al. Burnout and suicidal ideation among U.S. medical students. Ann Intern Med 2008;149:334-41.

29. Maslach C, Schaufeli WB, Leiter MP. Job burnout. Annu Rev Psychol 2001;52:397-422.

30. Lovibond SH, Lovibond PF. Manual for the Depression Anxiety Stress Scales. 2nd ed. Sydney, Australia: Psychology Foundation; 1995.

31. Gomez F. A guide to the depression, anxiety, and stress scale (DASS 21). https://jeanmartainnaturopath. com.au/wp-content/uploads/2016/10/Dass21.pdf. Accessed April 23, 2020.

32. Osman A, Wong JL, Bagge CL, Freedenthal S, Gutierrez PM, Lozano G. The Depression Anxiety Stress Scales-21 (DASS-21): further examination of dimensions, scale reliability, and correlates. J Clin Psychol 2012;68:1322-38.

33. Statistics Kingdom. Chi-square statistical power calculator. http://www.statskingdom.com/34test_ power_chi2.html. Accessed July 20, 2020.

34. Kim HY. Statistical notes for clinical researchers: chi-squared test and Fisher's exact test. Restor Dent Endod 2017;42:152-5.

35. Data Star, Inc. What every researcher should know about statistical significance. 2008. http://www. surveystar.com/startips/oct2008.pdf. Accessed November 11, 2020.

36. Stokes EK, Zambrano LD, Anderson KN, et al. Coronavirus disease 2019 case surveillance-
United States, January 22-May 30, 2020. MMWR Morb Mortal Wkly Rep 2020;69:759-65.

37. Rodriguez RM, Medak AJ, Baumann BM, et al. Academic emergency medicine physicians' anxiety levels, stressors, and potential stress mitigation measures during the acceleration phase of the COVID-19 pandemic. Acad Emerg Med 2020;27:700-7.

38. Pearman A, Hughes ML, Smith EL, Neupert SD. Mental health challenges of United States healthcare professionals during COVID-19. Front Psychol 2020;11:2065.

39. The LA. Green Center and the Primary Care Collaborative. Quick COVID-19 primary care survey: series 14. https://static1.squarespace.com/static/ $5 \mathrm{~d} 7 \mathrm{ff} 8184 \mathrm{cf0e} 01 \mathrm{e} 4566 \mathrm{cb} 02 / \mathrm{t} / 5$ eea695edc760012 fe9a1dea/1592420704960/C19+Series+14+National+ Executive+Summary.pdf. Accessed November 21, 2020.

40. Reynolds KA. Coronavirus: primary care physicians still feeling the stress of pandemic, survey finds. Medical Economics. https://www.medicaleconomics. com/view/coronavirus-primary-care-physicians-stillfeeling-stress-pandemic-survey-finds. Accessed November 21, 2020.

41. Dyrbye LN, Shanafelt TD. Physician burnout: a potential threat to successful health care reform. JAMA 2011 May 18;305:2009-10.

42. Ofei-Dodoo S, Kellerman R, Nilsen K, Nutting R, Lewis D. Family physicians' perceptions of electronic cigarettes in tobacco use counseling. J Am Board Fam Med 2017 Jul-Aug;30:448-59.

43. Mills K, Nilsen K. Kansas family physicians perceptions of parental vaccination hesitancy. Kansas Journal of Medicine 2020;13:248-59.

44. Ofei-Dodoo S, Wipperman J, Nutting R, Gilchrist $\mathrm{K}$, Kellerman R. Changes in family physicians' perceptions of electronic cigarettes in tobacco use counseling between 2016 and 2019. Kansas Journal of Medicine 2020;13:311-7. 
Appendix. How Family Physicians Are Responding to COVID-19 Questionnaire

1. Have you cared for presumptive or confirmed COVID-19 patients in your practice?

Yes No

2. How many presumptive or confirmed COVID19 patients have you treated?

3. Where have you treated COVID-19 patients? (Please check all that apply)

a. In office

b. At the hospital

c. Via telehealth

d. At the ER

e. Home visits

4. For each of the following statements, please check the box that most accurately reflects your response:

a. I feel burned out from my work since the COVID-19 outbreak.

b. I've become more calloused towards people since the COVID-19 outbreak.

The rating scale is as follows:

0 . Never

1. A few times a year

2. Once a month or less

3. A few times a month

4. Once a week

5. A few times a week

6. Every day

5. How are your patient volumes now, compared to before the pandemic?

a. I'm seeing more patients

b. I'm see fewer patients

c. I'm seeing about the same number of patients

6. Are you concerned that some of your patients may be foregoing routine or acute medical care because they are afraid of exposure to COVID-19 in an office or other clinical setting? Yes No

7. What is your level of personal stress as a result of the COVID-19 pandemic?

0 (No stress) 12345678910 (Extreme stress)

8. What was your level of stress prior to the COVID-19 pandemic?

0 (No stress) 12345678910 (Extreme stress)

9. What is your level of concern about personally being exposed to COVID-19 because of your work?

0 (Not concerned) 12345678910 (Very concerned)

10. What is your level of concern about exposing family members to COVID-19 because of your work?

0 (Not concerned) 12345678910 (Very concerned)
11. For each statement below, please indicate how you have been feeling during the past week:

a. I found it hard to wind down

b. I tended to over-react to situations

c. I felt that I was using a lot of nervous energy

d. I found myself getting agitated

e. I found it difficult to relax

f. I was intolerant of anything that kept me from getting on with what I was doing

g. I felt that I was rather touchy

h. I was aware of dryness of my mouth

i. I experienced breathing difficulty (eg, excessively rapid breathing, breathlessness in the absence of physical exertion)

j. I experienced trembling (eg, in the hands)

k. I was worried about situations in which I might panic and make a fool of myself

1. I felt I was close to panic

$\mathrm{m}$. I was aware of the action of my heart in the absence of physical exertion (eg, sense of heart rate increase, heart missing a beat)

n. I felt scared without any good reason

o. I couldn't seem to experience any positive feeling at all

p. I found it difficult to work up the initiative to do things

q. I felt that I had nothing to look forward to

r. I felt downhearted and blue

s. I was unable to become enthusiastic about anything

t. I felt I wasn't worth much as a person

u. I felt that life was meaningless

The rating scale is as follows:

0 . Did not apply to me at all

1. Applied to me to some degree, or some of the time

2. Applied to me to a considerable degree or a good part of time

3. Applied to me very much or most of the time

12. What is your gender?

$\square$ Male

Female

Prefer to not answer

Other (please specify)

13. What year were you born?

14. In which Kansas county do you primarily practice?

15. How many years have you been in practice since residency?

Thanks for participating! 